Estudios Románicos, Volumen 28, 2019, pp. 179-190

ISSN: 0210-491

eISSN: 1989-614X

DOI: https://doi.org/10.6018/ER/377841

\title{
DE LA MAREA GRANATE A LOS GILETS JAUNES: HUMÁNIMOS FRASEOLÓGICOS CROMÁTICOS
}

(From the marea granate to the gilets jaunes: chromatic phraseological humanyms)

\author{
Cristian Díaz Rodríguez \\ Universidad de Estrasburgo
}

\begin{abstract}
In this paper, we propose a study of Spanish and French phraseological units that denominate human entities (humanyms) whose structure follows this pattern: Noun + Chromatic Adjective: gilet jaune, casques bleus, camisas azules, marea granate. Considering the theories about phraseological meaning explicative model, proposed by L. Timofeeva $(2008,2012)$, and basing our analysis on the iconic models formulated by E. M. Iñesta and A. Pamies (2002), we propose a series of criteria that will permit to distinguish between nominal phraseological units used to qualify humans (idioms) and those that, on the contrary, create stable classes of referents (phraseological compounds). We will also analyze the role played by chromatic adjectives as a vector to carry the ethnocultural and idiosyncratic trace of the community of speakers that have created all these phraseological humanyms.
\end{abstract}

Keywords: Phraseology; Chromatism; Humanym; Idiom; Compound.

Resumen: En el presente artículo se propone un estudio de las unidades fraseológicas francesas y españolas que denominan entidades humanas (humánimos) y cuya estructura responde al esquema $\mathrm{N}+\mathrm{Adj}_{\text {cromático }}$ : gilet jaune, casques bleus, camisas azules, marea granate. Teniendo en cuenta las teorías propuestas por L. Timofeeva (2008, 2012) sobre el modelo explicativo del significado fraseológico, y tomando como base de análisis los modelos icónicos enunciados por E. M. Iñesta y A. Pamies (2002), se propone una serie de criterios que permiten distinguir las unidades fraseológicas nominales utilizadas para calificar a los humanos (locuciones nominales) de aquellas que, por el contrario, crean clases estables de referentes (compuestos fraseológicos). Asimismo, se

* Dirección para correspondencia: Cristian Díaz Rodríguez. Département de Langues Étrangères Appliquées, Le Patio, bureau 5131. Faculté des Langues - Université de Strasbourg. 22, rue René Descartes. F-67804 Strasbourg | Francia (cdiazrodriguez@unistra.fr). 
analiza el papel que desempeñan las lexías cromáticas a la hora de vehicular la huella etnocultural e idiosincrática de la comunidad lingüística que ha acuñado los diferentes humánimos fraseológicos estudiados.

Palabras clave: Fraseología; Cromatismo; Humánimo; Locución; Compuesto.

\section{Introducción}

A pesar de la aparente facilidad clasificatoria de los sustantivos que denominan entidades humanas, a los que nos referiremos de ahora en adelante como humánimos, lo cierto es que este subgrupo de nombres encierra, tal y como se señala en el descriptivo del proyecto NHUMA ${ }^{1}$, una serie de particularidades que dificultan la labor taxonómica:

De prime abord, ces noms ne semblent pas poser de problème majeur : ils désignent des entités concrètes, comptables et hétérogènes, dont l' « évidence extralinguistique » est telle que les classifications en usage ne semblent pas devoir être questionnées.

Un examen attentif des faits révèle pourtant un certain nombre de difficultés, culturelles (la bipartition humain vs non humain n'est pas la même dans toutes les langues), mais aussi ontologiques (les hiérarchies habituelles, du type une pomme est un fruit, s'appliquent difficilement aux entités humaines, cf. l'impossibilité de dire un plombier est un homme) et psychologiques (les catégories sociales renvoyant aux humains font l'objet de classifications hétérogènes multicritériées par les sujets plutôt que de taxonomies). (Descriptivo del Proyecto NHUMA)

El interés que despierta esta subclase léxica nominal queda patente en la reciente profusión de estudios que, barriendo todo el espectro lingüístico, y, por lo general, bajo un enfoque pluridisciplinar, han abordado los humánimos (entre otros, Mihatsch y Schnedecker 2015; Schnedecker y Mihatsch 2018; Schnedecker 2018). Sin embargo, la inmensa mayoría de los trabajos, cuando no la totalidad, se centran en el estudio de humánimos monoléxicos, haciendo abstracción, salvo raras excepciones, de aquellos de carácter poliléxico. La extensión del estudio de los humánimos a los polilexemas, es decir, a la esfera fraseológica lato sensu, es tanto más necesaria cuanto que el problema taxonómico afecta, en este caso concreto, a la propia identificación de humánimos fraseológicos, al tener que operar la distinción entre compuestos locucionales y locuciones nominales.

En este artículo nos centramos en un subgrupo de unidades fraseológicas (en adelante UF) caracterizadas por compartir un mismo patrón estructural: $\mathrm{N}+\operatorname{Adj}_{\text {cromático }}$ (=AdjCro). Esta elección se justifica por su alta productividad a la hora de crear UF

1 El proyecto NHUMA (https://nomsdhumains.weebly.com/ [consultado el 01/02/2019]) tiene por objetivo el estudio y la clasificación de los humánimos bajo un enfoque que va más allá de la semántica léxica. De hecho, en el proyecto se integran tanto semantistas, como morfólogos, lingüistas informáticos, traductólogos..., cuyas lenguas de estudio no sabrían limitarse al francés, de manera que las reflexiones se enriquecen con las observaciones realizadas en las diferentes lenguas románicas, germánicas, eslavas, etc. 
que designan entidades humanas. Así, por ejemplo, en los últimos meses, venimos asistiendo, en francés europeo, al surgimiento de neologismos poliléxicos empleados para dar nombre a diferentes movimientos sociales que hacen del color de un elemento que los representa metonímicamente su carta de presentación o, si se prefiere, de denominación: gilets jaunes, gyros bleus, stylo rouges ${ }^{2}$, etc. Un fenómeno parecido, al menos en lo que se refiere al patrón estructural, $\mathrm{N}+\mathrm{AdjCro}$, se produjo igualmente en el español europeo a finales del primer mandato del gobierno de Mariano Rajoy: marea verde, marea blanca, marea violeta, marea azul, marea negra, marea granate 3 , etc. A pesar del carácter efímero de algunas de estas denominaciones, en cierta medida condicionadas por el devenir mismo de los movimientos sociales a los que designan, lo cierto es que la elección de cada uno de los colores presenta un interés especial desde el punto de vista lingüístico, pues la lexía cromática puede convertirse eventualmente en vector vehiculante de la huella etnolingüística e idiosincrática de la comunidad lingüística que crea dichos humánimos. En aras de garantizar la convencionalización, e incluso la institucionalización de las UF que aquí analizamos, hemos construido nuestro corpus de estudio a partir de la extracción de todas las UF nominales referidas a entidades humanas incluidas en cuatro diccionarios con motor de búsqueda: el Trésor de la langue française informatisé (TLFi) y el Petit Robert (PR) para el francés, y el Diccionario de la Lengua Española $(D E L)$ y el Diccionario inverso de la RAE (DiRAE) para el español.

Tras hacer un breve repaso sobre la capacidad denominativa de las lexías cromáticas, ofreceremos, en una segunda parte, una serie de características ligadas al significado fraseológico, que permiten distinguir netamente las UF nominales que cumplen una función calificadora (locuciones), y que, por lo tanto, no deben considerarse humánimos fraseológicos, de aquellas UF que designan clases estables de referentes (compuestos). En una tercera parte presentaremos los modelos icónicos más recurrentes a la hora de crear humánimos fraseológicos. Nos interesaremos igualmente por el papel que desempeña el AdjCro en la construcción del significado fraseológico de la combinación.

\section{Funciones de las lexías cromáticas}

Además de la importancia que tienen los estímulos cromáticos en la comunicación visual, los colores permiten expresar cualidades de realidades ontológicas mediante la calificación de referentes: veste rouge, trousse noire, coche verde, pantalón azul. No en vano, la mayoría de las gramáticas presentan los colores como prototipos de adjetivos

2 Respectivamente: a) los chalecos amarillos que portan los manifestantes que protestan contra la pérdida de poder adquisitivo durante el mandato del presidente Macron; b) los faros autogiros azules de las sirenas, que representan al colectivo de policías, quienes exigen una mejora de sus condiciones laborales y c) el colectivo de profesores, designados mediante los bolígrafos rojos, que, de esta forma, expresan la rabia que sienten contra la reforma del sistema educativo francés.

3 Movimientos que defendían respectivamente: a) la gratuidad y la universalidad de la educación; b) la oposición a la privatización del sistema sanitario; c) el hacer prevalecer los derechos de las mujeres; d) la oposición a la privatización de la gestión del agua en Madrid; e) al colectivo funcionarial que había servido como chivo expiatorio de las políticas de austeridad y f) la defensa, bajo el color del pasaporte, del derecho a la "no expatriación" de todos los jóvenes que habían tenido que abandonar España para poder labrarse un futuro profesional en el extranjero. 
calificativos. Sin embargo, esta consideración debería ser cuestionada, puesto que los AdjCro también permiten estructurar el mundo referencial creando clases estables de elementos, es decir, funcionando como adjetivos clasificadores ${ }^{4}$ (Girardin 2005): poivron rouge, ours blanc, chocolate negro, ballena azul. La importancia de los colores no podría reducirse en ningún caso a la mera caracterización física. Muy al contrario, estos vehiculan igualmente una serie de valores idiosincráticos y axiológicos que constituyen la huella indeleble de una comunidad de hablantes determinada (Pastoureau 1992), de manera que las lexías cromáticas no solo califican o clasifican el mundo sensible sino también realidades o conceptos desprovistos de corporeidad: un mardi noir, une énergie verte, une âme blanche, un humor negro, una vida de color de rosa, una vida gris.

\subsection{El color como calificador humano}

La distinción que acabamos de ver, color ontológico vs color idiosincrático, también es operante en el caso en el que el elemento calificado por el AdjCro sea una entidad humana. Ora la lexía cromática expresa ciertamente un color perceptible, que describe la pigmentación de la piel humana (1-2), generalmente de tipo sintomático (3-6). Ora el semantismo expresado por el $\mathrm{AdjCro}$ se le ha atribuido mediante un proceso de convencionalización (7-10).

(1) Espero ponerme negra de aquí a que acabe el verano.

(2) Il ne bronze pas et reste blanc comme un cachet d'aspirine.

(3) Supimos que había pasado miedo porque salió superpálido, casi amarillo.

(4) Dès qu'il fait un peu froid, la peau de mes mains, de mes genoux et de mon front devient violette, presque bleue.

(5) El niño lleva mucho tiempo en el agua, se está empezando a poner morado.

(6) Le visage de M. Girouard est devenu très pâle, puis tout rouge quand celui-ci s'est mis à essayer de recracher la nourriture qui l'étouffait.

(7) Se veía que el tutor de la clase de $1^{\circ} B$ se estaba poniendo verde de envidia, al ver lo bien que se expresaban los alumnos de $1^{\circ} \mathrm{A}$.

(8) Lorsque les enfants du voisinage verront votre enfant sur cette machine, colorée et moderne-trike, ils seront verts d'envie.

(9) La primera vez que fui a un buffet me puse morado.

(10) Une personne amère est une personne grise et aigrie.

\subsection{El color como clasificador humano: humánimos cromáticos}

Si hasta el momento hemos visto que los AdjCro pueden calificar las entidades humanas, lo cierto es que las lexías cromáticas, en tanto que substantivos, también pueden designar subgrupos de humanos clasificándolos en función del color de su tez, es decir,

$4 \quad$ Hemos decidido omitir las funciones intensificadoras (Grossmann y Tutin 2005): peur bleue, verte réprimande, pena negra, verde de envidia, y relacionales: quartier blanc, música negra, desempeñadas eventualmente por AdjCro, pues carecen de interés para nuestro estudio sobre los humánimos. 
estableciendo una taxonomía basada en un criterio cutáneo o dérmico: les Blancs |los blancos, les Noirs | los negros ${ }^{5}$, les gens de couleur | la gente de color, les jaunes, les peau rouges $\mid$ los pieles-roja, etc. De igual modo, se puede realizar una clasificación cromática de los humanos que responda a un criterio para-dérmico, al asociar, por convención, ciertos colectivos humanos a un color representativo: el de una bandera (los rojos: bandera comunista), el de una ideología (les Verts: ecología), el de un comportamiento (les jaunes: traición) o simplemente el de una segunda piel, a saber, la vestimenta (los grises: color del uniforme de los policías durante la dictadura franquista). En este último sentido, habría que destacar la función denominativa y taxonómica de los colores en el ámbito deportivo, pues metonímicamente es posible nombrar al equipo a través del color de su equipación: Allez les Bleus! (les Bleus = la selección francesa); Allez les Verts! (les Verts $=$ el equipo Saint-Etienne); Los blancos anotaron 3 goles frente al Betis (los blancos = el Real Madrid); La Roja ha caído en cuartos de final (La Roja ${ }^{6}=$ la selección española). La designación por medio de una combinación de colores también es posible: los albicelestes (la selección argentina), los blaugranas (los jugadores del F. C. Barcelona), etc. Estos colores son tanto más idiomáticos cuanto que pueden variar de un país a otro o, incluso, de una región a otra en el seno de una misma comunidad de hablantes.

(11) a. Coupe de la Ligue: "Merci les Bleus!" (DNA) [les Bleus = Sporting de Strasbourg]

b. Les Bleus 2018: au cour de l'épopée russe (Le Monde) [les Bleus = la selección francesa masculina de fútbol]

(12) Derrota de los blanquiazules en casa [blanquiazules = CD Tenerife, Málaga, etc.]

Además, estos humánimos cromáticos están tan institucionalizados que su uso denominativo sigue siendo válido aun cuando los jugadores saltan al terreno de juego con su segunda o tercera equipación, y, por lo tanto, sin vestir los colores con los que se los denomina.

\section{De las unidades fraseológicas a los humánimos fraseológicos}

Podemos definir las UF como secuencias poliléxicas constituidas de al menos dos palabras ligadas categorialmente, cuya disposición estructural puede ser contigua o no, caracterizadas por cierto grado de fijación, fenómeno polifactorial que se puede declinar principalmente en tres vertientes: la fijación formal o estructural, la fijación del con-

5 Como señalábamos anteriormente, estos humánimos cromáticos pueden llegar a dar lugar a adjetivos relacionales: música negra, quartiers blancs. Nótese lo peculiar de estos adjetivos cromáticos, pues, a pesar de su carácter relacional (negra $=$ de los negros; blancs = des Blancs), carecen de rasgos morfológicos propios (cfr. francés $=$ de Francia, doctoral $=$ de doctorado, universitaire $=$ de l'université, ministeriel $=$ du Ministère).

6 Contrariamente a lo que sucede con les Bleus, aplicable a todos los jugadores de cualquier selección francesa, independientemente del deporte practicado, la Roja solo hace referencia a la selección absoluta masculina de fútbol, de ahí que no pueda considerarse exactamente un humánimo, sino más bien un caso especial de antropónimo, objeto de estudio, pues, de la onomástica. 
tenido, y la fijación socio-discursiva. La fijación formal da cuenta de las características relacionadas con el nivel morfosintáctico y léxico -esencialmente, bloqueo de las operaciones transformacionales (13), cierre paradigmático (14) e inalterabilidad del orden de los constituyentes (15). Por su parte, la fijación del contenido contempla las características de los niveles semántico y pragmático - no composicionalidad [gilets jaunes $\neq$ 'gilets' + 'jaune']. La fijación socio-discursiva también afecta el nivel pragmático, pero se centra en las imposiciones/restricciones discursivas: Allez les Bleus!

(13) a. L'incroyable projet de Bercy qui va rendre vert les gilets jaunes / *les gilets dont la couleur est jaune.

b. Las mareas granate / *Las mareas cuyo color es granate de toda Francia se congregarán mañana en París.

(14) a. L'incroyable projet de Bercy qui va rendre vert les *tricots jaunes / les *gilets jaune fluo.

b. Las mareas granate /*Las mareas burdeos / *Las olas granate de toda Francia se congregarán mañana en París.

(15) a. L'incroyable projet de Bercy qui va rendre vert *les jaunes gilets.

b. Las mareas granate / *Las granate mareas de toda Francia se congregarán mañana en París.

Contrariamente a la fijación formal, cuya manifestación es escalar, el enriquecimiento semántico-pragmático del significado global constituye una condición sine qua non para que se pueda hablar de UF. De ahí que el significado fraseológico nunca pueda ser composicional (Díaz Rodríguez 2017a), pues, además de la componente denotativa, incluirá inferencias a diferentes niveles de convencionalización (Timofeeva 2008 , 2012). Observamos que, en el caso de gilet jaune, además de la reinterpretación semántica de los constituyentes de la UF, el significado global incluye un enriquecimiento \{'contra el aumento del precio de la gasolina y, de manera, más general, contra la pérdida del poder adquisitivo, caracterizada por llevar un chaleco amarillo para mostrar su adhesión al movimiento ciudadano homónimo'\}, lo que permitiría, llegado el caso, su supervivenvia frente a otros casi-sinónimos monoléxicos como manifestante o huelguista.

\subsection{El significado fraseológico como criterio discriminante}

Si tomamos como referencia el modelo explicativo del significado fraseológico (véase Tabla 1) propuesto por L. Timofeeva $(2008,2012)$, podemos constatar que, además del significado propiamente semántico-denotativo, cada uno de los bloques que define el significado fraseológico incluye un enriquecimiento que se traduce por una evaluación cuantitativa, cualitativa o axiológica del bloque denotativo, de manera que el significado fraseológico global se posicionará sobre una escala que establece como punto de referencia un punto neutro, a saber, la información semántico-denotativa, generalmente lexicalizada mediante una lexía simple. 
De la marea granate a los gilets jaunes: humánimos fraseológicos cromáticos

\begin{tabular}{|l|l|}
\hline \multicolumn{1}{|c|}{ Bloques } & \multicolumn{1}{c|}{ PRÍNCIPE AZUL } \\
\hline Denotativo & Hombre ideal \\
\hline Motivacional & $\begin{array}{l}\text { Imagen heredada de los cuentos tradicionales, recopilados durante el } \\
\text { Romanticismo (cfr. libro azul de Nerval) en las que se concentran las } \\
\text { virtudes del hombre ideal en la figura de un príncipe. }\end{array}$ \\
\hline $\begin{array}{l}\text { Evaluativo- } \\
\text { racionalizado }\end{array}$ & $\begin{array}{l}\text { Cuantitativo: neutro } \\
\text { Cualitativo: positivo }\end{array}$ \\
\hline $\begin{array}{l}\text { Evaluativo } \\
\text { emotivo-emocional }\end{array}$ & $\begin{array}{l}\text { La imagen transporta al mundo onírico y fantástico, por lo que insiste } \\
\text { en la idea de la imposibilidad de encontrarlo en el mundo real. }\end{array}$ \\
\hline Estilístico & Estándar \\
\hline Gramatical & Locución nominal \\
\hline
\end{tabular}

Tabla 1: Primer grado de concreción del modelo explicativo del significado fraseológico.

En función de cómo se construye el significado fraseológico, podemos distinguir dos tipos de comportamientos: el comportamiento locucional y el comportamiento colocacional. El primero describe a las UF cuyo significado es el resultado de la reinterpretación semántica global de los constituyentes, de ahí que su significado sea unitario ${ }^{7}$ : bestia negra, boinas verdes, talon rouge, chien jaunes, etc. El significado de las UF con comportamiento colocacional es de tipo aditivo, pues es el resultado de la suma de los significados de los componentes, eventualmente reinterpretados de manera individual (polisemia condicionada), más el enriquecimiento semántico-pragmático inherente a toda UF: enfant bleu, armée blanche, guardia roja, marea \{verde, azul, blanca, violeta, granate, negra\}. Observamos que, en este caso, el elemento autosemántico designa, per se, una entidad humana (enfant, armée, guardia, marea $^{8}$ ), mientras que el elemento sinsemántico, además de aportar una noción cromática, incluye una serie de informaciones asignadas mediante un proceso de convencionalización. En este artículo restringiremos nuestro estudio a las UF de comportamiento locucional pues son las que, en principio, revisten de un mayor interés.

\subsection{Locuciones nominales vs compuestos locucionales}

Con independencia de cuál sea su comportamiento fraseológico, todas las UF nominales cumplen una función, ya sea calificadora (calificativa, intensificadora, evalua-

$7 \quad$ Es posible que los significados de los componentes de una UF locucional puedan ser integrados en el significado global, pero, en ese caso lo harán en tanto que semas y no de manera composicional. Así, aun cuando los significados de casco y de azul participan en la definición de casques bleus $\mid$ cascos azules 'militares de la ONU en misión de paz que llevan un casco de color azul', estos lo hacen como semas.

8 Bien que l'AdjChr qui accompagne la lexie marée dans cette deuxième série ait eu une justification métonymique à l'origine (la couleur des t-shirts des manifestants), de nos jours, la couleur n'est qu'une sorte de vestige d'une conventionnalisation idiosyncrasique. De fait, ces noms composés collocationnels sont devenus des appellations utilisées pour parler de l'ensemble d'actions organisées au sein d'un collectif travaillant en faveur des objectifs incarnés par les différentes couleurs. Cela implique la reconnaissance d'une nouvelle resémantisation du terme marée qui passe d'un 'cortège' à un 'ensemble d'actions de mobilisation en faveur de certains objectifs'(Díaz Rodríguez 2017b: 236). 
tiva, etc.) o clasificadora. Las primeras coinciden con lo que tradicionalmente se ha llamado locución nominal. El bloque semántico-denotativo de estas UF no se refiere a ninguna clase estable de referentes, de ahí que muchas veces se pueda aplicar a más de un tipo de elemento (16-17). Incluso, en el caso de las locuciones nominales que gozan de mayor estabilidad denotativa, se trata de una designación evenemencial, muy subjetiva y, por consiguiente, caracterizada por una alta adjetividad (Salles 2004), de ahí que algunos autores asemejen su comportamiento al de los adjetivos (García-Page Sánchez 2012): príncipe azul, pisaverde, cordon-bleu, chat noir.

(16) Le latin / Mme Schneider / Le Barça est sa bête noire.

(17) a. El Tenerife es el farolillo rojo de la clasificación.

b. Si no estudias un poco más, acabarás siendo el farolillo rojo de la clase.

Las UF de función clasificadora, por su parte, identifican los referentes con un grupo determinado, en función de una serie de rasgos definitorios inherentes y esenciales, que los diferencian de otros grupos o clases de referentes. Nombran, pues, clases estables a las que la pertenencia de un individuo está garantizada con independencia de las circunstancias enunciativas (no evenemenciales) y de las fluctuaciones que el elemento evaluado pueda presentar con respecto al prototipo que define la clase. Se trata, por lo tanto, de una denominación estable y, por lo general, objetiva. Se desprende de lo dicho que solo las UF de función clasificadora, es decir, los compuestos locucionales, que designen entidades humanas pueden ser considerados humánimos fraseológicos ${ }^{9}$. Ahora bien, si para algunos casos fronterizos la dicotomía calificador vs clasificador no es totalmente operante ${ }^{10}$, esta es tanto menos sencilla cuanto que la mayoría de humánimos fraseológicos construidos con una lexía cromática designan, como se verá ulteriormente, gentilicios o profesiones, es decir, categorías que gozan, por su propia naturaleza, de una alta adjetividad. Aun con todo, se muestran a continuación las UF nominales prototípicamente calificadoras, es decir, las locuciones nominales que contienen una lexía cromática y cuyo referente podría ser una entidad humana ${ }^{11}$ : cordon-bleu, aigle blanc, avaleur de pois gris, barbe-bleu, bas-bleu, bête noire| bestia negra", bec-jaune, blanc bec, bouffeur / mangeur / chiqueur de blanc, boyau rouge, broyeur de noir, chat noir ${ }^{\#}$, chevalier blanc | caballero blanco ${ }^{\#}$, dos d'azur, dos vert, éminence grise | cerebro gris, fleur bleue, foie-blanc, fruit vert, gant jaune, lanterne rouge $\mid$ farolillo rojo ${ }^{\#}$, merle blanc $\mid$ mirlo blanco, mouton noir | oveja negra, oie blanche, oiseau bleu, peau-rouge, talon rouge, vert-galant, veuve noire |viuda negra, bocanegra, príncipe azul, garbanzo negro, mosca blanca, mano negra ${ }^{\#}$, indiano de hilo negro, pisaverde, bestia parda.

9 En este sentido existen grandes discordancias entre la lexicografía francesa y su homóloga española, pues allí donde la primera suele hablar, a nuestro juicio con razón, de locución nominal (es el caso, entre otros de las UF bête noire | bestia negra y lanterne rouge | farolillo rojo, empleadas en nuestros ejemplos), la lexicografía española prefiere emplear el término compuesto nominal.

10 La diferencia entre locuciones y compuestos, dada la continuidad existente entre calificativo y clasificador, puede resultar en algunos casos poco operante. Por eso, debemos hablar en términos de UF prototípicamente calificadoras o clasificadoras.

11 Hemos señalado con el símbolo '\#’ aquellas que son aplicables, además, a otros referentes no humanos. 


\section{Humánimos fraseológicos abigarrados}

La teoría de los modelos icónicos y de las archimetáforas propuesta por E. M. Iñesta y A. Pamies (2002) enuncia que las UF creadas mediante metaforización para expresar conceptos en un dominio meta no son sino casos particulares (metáforas particulares) de una serie más restringida de metáforas generales o archimetáforas, creadas a partir de la combinación (modelos icónicos) de un conjunto muy limitado de dominios fuente ${ }^{12}$. Así, por ejemplo, para representar el dominio meta miedo, se pueden seleccionar los dominios fuente [CUERPO] y [MOVIMIENTO], para crear el modelo icónico [CUERPO] + [MOVIMIENTO]. En el primer nivel de concreción de este modelo se encontrarán las diferentes archimetáforas (el miedo es un movimiento corporal hacia arriba / abajo / adentro / afuera, etc.) que darán lugar, posteriormente, a metáforas particulares: ponerse los pelos de punta, cagarse encima, estar invadido por el miedo...

En principio, los modelos icónicos solo fueron concebidos para explicar la transferencia semántica que se produce en las locuciones, caracterizadas por una metaforización entre diferentes dominios (inter-dominio). Sin embargo, en los compuestos locucionales, a priori excluidos de esta modelización, se comprueba que la transferencia semántica que se produce es de tipo intra-dominio. Por eso, en el caso de los humánimos fraseológicos, hablaremos en términos de archimetonimia y de metonimias particulares.

\subsection{De los modelos icónicos a las metonimias particulares}

Son varios los modelos icónicos que sirven para crear archimetonimias que designan humanos. Una de las más productivas sería la definida por el modelo icónico [CUERPo] + [COLOR] según la cual 'se utiliza una parte del cuerpo en lugar de la persona a la que pertenece'. En nuestro corpus de estudio encontramos las siguientes metonimias particulares de esta archimetonimia: barbe grise, bleue bitte, cul-blanc, gueules noires, menton bleu, pied-noir, tête grise, culipardo, culiblanco

Otro modelo icónico posible vendría representado por la combinación de dominios fuente [OBJETOS] + [COLOR], de manera que se nombraría al 'instrumento de trabajo en lugar de la persona que lo utiliza': escopeta negra, libro verde, stylos rouges, gyros bleus. Como ya habíamos señalado, el AdjCro no siempre refleja un color ontológico, sino que su elección puede obedecer eventualmente a una razón cultural, como ocurre en el caso de stylo rouge: stylo rouge + rouge de colère.

Existe un modelo icónico de creación de humánimos en el que la lexía cromática, a pesar de ser el fruto de un proceso de convencionalización, sí que refleja un color ontológico ${ }^{13}$. Se trata del modelo icónico [COLOR] + [VestimeNTA], cuya concreción en

12 La lista de los dominios fuente primitivos correspondería grosso modo a los conceptos universales y primitivos propuestos por A. Wierzbicka (2000): [persona - cuerpo], [espacio (localización o cantidad)], [tiempo], [movimiento], etc., a los que M. E. Iñesta y A. Pamies habrían añadido: animal, vegetal, objeto, fenómeno atmosférico y necesidades fisiológicas.

13 Puede suceder que la elección de un determinado color esconda detrás de su naturaleza ontológica una razón idiosincrática. Así, por ejemplo, aunque los gilets jaunes llevan efectivamente un chaleco de color amarillo, esta elección no es anodina, pues, como bien sabemos, el amarillo representa, para los franceses, el color de la traición y de la falsedad (cfr. syndicat jaune, rire jaune, jaune cocu, etc), lo que podría entenderse como una acusación directa al presidente Macron por haber traicionado al pueblo, que depositó su confianza en él en las últimas elecciones. 
metonimias particulares ('la vestimenta en lugar de la persona que la lleva') da lugar a numerosos humánimos, que nombran subclases estables de personas: bas violet, bérets verts $\mid$ boinas verdes, blouses blanches, blouson noir, bonnet rouge, casques bleus $\mid$ cascos azules, ceinture noire | cinturón negro, col blanc, col bleu, chemise noire | camisas negras, chemise rouge | camisas rojas, chemises brunes | camisas marrones, cordon-bleu, foulard rouge, gant jaune, habit (à palmes) vert(es), habits rouges, bérets rouges, maillot jaune, maillot rose, maillot doré, maillot rouge, pompon rouge, robe noire, ruban rouge, tablier blanc, uniforme vert-de-gris, camisa azul.

Como se desprende de la gran cantidad de humánimos que acabamos de enumerar, debemos destacar la importancia de las vestimentas como elementos fundamentales a la hora de crear compuestos locucionales que designan humanos. Este dominio fuente no goza, por lo general, de autonomía categorial. De hecho, los elementos de este grupo se suelen amalgamar en otros dominios fuentes como CUERPO, OBJETOS o incluso CONVENCIÓn SOCiAL. Sin embargo, en el caso preciso de los compuestos locucionales, nos parece pertinente la creación de un dominio fuente independiente: VESTIMENTA.

Como hemos venido señalando, la predilección por la metonimia frente a la metáfora representaría una característica distintiva de los compuestos locucionales con respecto a las locuciones nominales. Sin embargo, en nuestro corpus también encontramos humánimos en los que se combinan simultáneamente ambos métodos reinterpretativos. Es el caso, por ejemplo, de los compuestos chien jaune, moscas verdes y diables bleus, en los que los nombres - chien, moscas y diables- experimentan una reinterpretación metafórica inter-dominio (ANIMAL/IMAGEN SOCIOCULTURAL $\rightarrow$ HUMANO), mientras que los AdjCro se eligen por metonimia a través de una relación intra-dominio: la substitución de la profesión por el color, convencionalmente atribuido, del uniforme característico, contradiciendo, pues, en cierta medida, eso de que el hábito no hace al monje.

\section{Conclusiones}

Tras analizar los datos de nuestro estudio debemos señalar, en primer lugar, que el francés es más productivo que el español a la hora de crear humánimos cromáticos locucionales. Tal y como habíamos anticipado, estas UF designan, sobre todo, gentilicios, nombres de profesiones y de afiliación política, resultado nada sorprendente, dado el valor semiótico e identitario de los colores. En este sentido, debemos señalar que los colores más utilizados corresponden a los no-colores (blanco y negro) y a los colores primarios-luz (rojo, azul y verde). Esta alta productividad en la creación de humánimos se justifica por el hecho de que coinciden con los colores con mayor carga idiosincrática y etnocultural. En lo referido a los dominios fuente en coalescencia con el color, debemos destacar el papel primordial de las vestimentas, así como de las partes del cuerpo. Por último, debemos subrayar el hecho de que son las vestimentas que cubren la parte superior del cuerpo las que abundan en nuestro corpus, seguidas de los sombreros/tocados. La visibilidad de la parte implicada, con respecto a las vestimentas que cubren la parte inferior del cuerpo o los zapatos, podría explicar esta predilección a la hora de ser elegida como elemento designador. 
En cuanto al enriquecimiento semántico-pragmático que incluyen estos humánimos fraseológicos, baste decir que, en el caso de los compuestos locucionales, dada su construcción ontológica a partir de una imagen subyacente, gozan de una mayor expresividad. A nivel evaluativo, solo los humánimos que sirven como denominación secundaria pueden incluir matices que evalúen el significado semántico-denotativo neutro. El objetivo que se persigue mediante su creación se declina en varias vertientes: peyorativo, laudativo, eufemístico, cómico, deshumanizador, cosificador, etc. Sin embargo, desde un punto de vista relacional, el uso de un humánimo fraseológico, en lugar de otro de tipo monoléxico, puede suponer una especie de intensificación del peso del locutor con respecto a lo que dice o a su interlocutor. Por último, cabe señalar que la supervivencia de un humánimo fraseológico puede justificarse por razones de variación diastrática o diafásica.

\section{BIBLIOGRAFÍA}

$[\mathrm{DEL}]=$ Diccionario de la lengua española . REAL ACADEMIA ESPAÑOLA, 24a edición, España, [en línea: http://rae.es/, consultado el 01/02/2019].

$[$ DiRAE $]=$ Diccionario inverso de Real Academia Española . RODRÍGUEZ ALBERICH, Gabriel y REAL ACADEMIA ESPAÑOLA, [en línea: http://dirae. es, consultado el 01/02/2019].

DÍAZ RODRÍGUEZ, Cristian (2017a): Étude contrastive français-espagnol des unités phraséologiques contenant une lexie chromatique. Tesis doctoral, Universidad de Estrasburgo - Universidad de La Laguna.

(2017b): "L'eau : inodore, incolore et insipide ? Un mensonge phraséologiquement inacceptable", LÓPEZ SANTIAGO, Mercedes et al. (eds.) Palabras e imaginarios del agua. Valencia: Editorial de la Universidad Politécnica de Valencia : 227-237.

GARCÍA-PAGE SÁNCHEZ, Mario (2012): "Pourquoi “un perrito caliente' n'est pas la même chose qu'una 'patata caliente'? Du composé à la locution nominale", BLANCO, Xavier; FUENTE, Sandrine y MEJRI, Salah (éds.), Les locutions nominales en langue générale. Francfort / Bellaterra: Servei de Publicacions de la Universitat Autònoma de Barcelona: 79-107.

GIRARDIN, Charlotte (2005): "Les classifieurs: une sous-classe d'adjectifs non prédicatifs". Cahiers de Lexicologie. Vol. 86: 59-70.

GROSSMANN, Francis y TUTIN, Agnès (2005): "Joie profonde, affreuse tristesse, parfait bonheur. Sur la prédicativité, des adjectifs intensifiant certains noms d'émotions". Cahiers de Lexicologie. Vol. 86: 1-18.

IÑESTA MENA, Eva María y PAMIES BERTRÁN, Antonio (2002): Fraseología y metáfora: aspectos tipológicos y cognitivos. Granada: Granada Lingvistica.

MIHATSCH, Wiltrud y SCHNEDECKER, Catherine (éds.) (2015): Les noms d'humains. Une catégorie à part? Stuttgart: Franz-Steiner Verlag, Collection Zeitschrift für französische Sprache und Literatur, vol. 40. 
PASTOUREAU, Michel (1992): Dictionnaire des couleurs de notre temps. Paris: Bonneton.

$[\mathrm{PR}]=$ Nouveau Petit Robert de la langue française (2018). Paris: Dictionnaires Le Robert.

SALLES, Mathilde (2004): "Adjectif et adjectivité ou comment un substantif peut être plus adjectif qu'un adjectif", L'Information Grammaticale. Vol. 103 / 1: 7-12.

SCHNEDECKER, Catherine (coord.) (2018): Dire l'humain. Les noms généraux dénotant les humains, Linx, 76.

SCHNEDECKER, Catherine y MIHATSCH, Wiltrud (éds.) (2018): Les noms d'humains -Théorie, méthodologie, classification Nouvelles approches en sémantique lexicale. Berlin: Mouton de Gruyter.

TIMOFEEVA, Larissa (2008): Acerca de los aspectos traductológicos de la fraseología española. Tesis doctoral, Universidad de Alicante.

(2012): El significado fraseológico. En torno a un modelo explicativo y aplicado. Liceus: Madrid.

$[\mathrm{TLFi}]=$ Trésor de la Langue Française Informatisé, CNRS, ATILF (Analyse et traitement informatique de la langue française), UMR CNRS-Université Nancy 2 [http://atilf.atilf.fr/frantext.htm, consultado el 12/01/2019].

WIERZBICKA, Anna (2000): "Primitivos semánticos y universales léxicos: teoría y algunos ejemplos", PAMIES BERTRÁN, Antonio y LUQUE DURÁN, Juan de Dios (coords.) Trabajos de lexicología y fraseología contrastivas. Granada: Método Ediciones: 1-28.

\section{PERFIL ACADÉMICO Y PROFESIONAL}

Cristian Díaz Rodríguez es doctor en Ciencias del Lenguaje-Estudios Filológicos por las Universidades de Estrasburgo y La Laguna. Actualmente trabaja como Profesor Ayudante Doctor en el Departamento de Lenguas Aplicadas de la Facultad de Lenguas de la Universidad de Estrasburgo. Su investigación se centra en el ámbito de la fraseología, teórica y aplicada, en la fraseografía bilingüe, la fraseodidáctica y la traducción.

Fecha de recepción: 14/05/2019

Fecha de aceptación: 08/07/2019 